\title{
An implementation of the NiftyRec medical imaging library for PIXE- \\ Tomography reconstruction
}

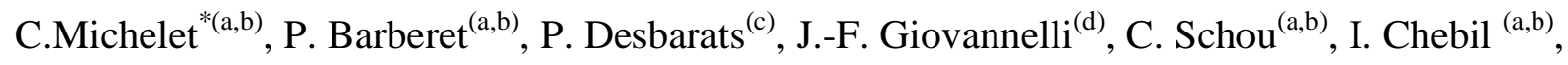

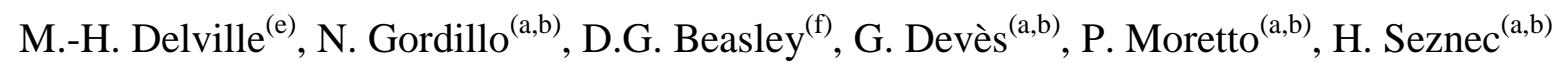

${ }^{a}$ Univ. Bordeaux, CENBG, UMR 5797, F-33170 Gradignan, France

${ }^{b}$ CNRS, IN2P3, CENBG, UMR 5797, F-33170 Gradignan, France

${ }^{c}$ Laboratoire Bordelais de Recherche en Informatique (LaBRI, UMR5800) Univ. Bordeaux, 351 Cours de la Libération, 33405 Talence cedex, France

${ }^{d}$ Intégration du Matériau au Système (IMS, UMR5218), Univ. Bordeaux, 351 Cours de la Libération, 33405 Talence, France

e Institut de Chimie de la Matière Condensée de Bordeaux (ICMCB, UPR9048) CNRS, Université de Bordeaux, 87 avenue du Dr. A. Schweitzer, Pessac, F-33608, France

${ }^{f}$ Center of Medical Image Computing (CMIC), Department of Medical Physics \& Bioengineering, University College London, Gower Street, London, WC1E 6BT, United Kingdom

\section{E-mail addresses:}

Claire MICHELET michelet@cenbg.in2p3.fr

Philippe BARBERET barberet@ cenbg.in2p3.fr

Pascal DESBARATS pascal.desbarats@labri.fr

Jean-François GIOVANNELLI giova@ims-bordeaux.fr

Caroline SCHOU caroline.schou@numericable.fr

Imen CHEBIL imen.chebil17@gmail.com

Marie-Hélène DELVILLE delville@icmcb-bordeaux.cnrs.fr 
Nuria GORDILLO nuri.gordillo@gmail.com

Daniel BEASLEY d.beasley@ucl.ac.uk

Guillaume DEVES deves@cenbg.in2p3.fr

Philippe MORETTO moretto@cenbg.in2p3.fr

Hervé SEZNEC seznech@cenbg.in2p3.fr

*Corresponding author. Tel.: +33 5571208 98; fax.: +33 557120801 .

E-mail address: michelet@ cenbg.in2p3.fr (C. Michelet).

Keywords:

PIXE tomography

Quantitative imaging

Filtered backprojection

MLEM

OSEM

Image J

Caenorhabditis elegans

\begin{abstract}
A new development of the TomoRebuild software package is presented, including "thick sample" correction for non linear X-ray production (NLXP) and X-ray absorption (XA). As in the previous versions, C++ programming with standard libraries was used for easier
\end{abstract}


portability. Data reduction requires different steps which may be run either from a command line instruction or via a user friendly interface, developed as a portable Java plugin in ImageJ. All experimental and reconstruction parameters can be easily modified, either directly in the ASCII parameter files or via the ImageJ interface. A detailed user guide in English is provided. Sinograms and final reconstructed images are generated in usual binary formats that can be read by most public domain graphic softwares. New MLEM and OSEM methods are proposed, using optimized methods from the NiftyRec medical imaging library. An overview of the different medical imaging methods that have been used for ion beam microtomography applications is presented. In TomoRebuild, PIXET data reduction is performed for each chemical element independently and separately from STIMT, except for two steps where the fusion of STIMT and PIXET data is required: the calculation of the correction matrix and the normalization of PIXET data to obtain mass fraction distributions. Correction matrices for NLXP and XA are calculated using procedures extracted from the DISRA code, taking into account a large X-ray detection solid angle. For this, the 3D STIMT mass density distribution is used, considering a homogeneous global composition. A first example of PIXET experiment using two detectors is presented. Reconstruction results are compared and found in good agreement between different codes: FBP, NiftyRec MLEM and OSEM of the TomoRebuild software package, the original DISRA, its accelerated version provided in JPIXET and the accelerated MLEM version of JPIXET, with or without correction.

\section{Introduction}

The reconstruction of ion beam tomography data raises a methodological challenge: the fusion of two data sets, coming from two different detectors and generally recorded in different experimental conditions [1]. The first data set comes from transmission tomography 
(Scanning Transmission Ion Microscopy Tomography, STIMT): the transmitted energy of the ion beam, which is directly related to the sample thickness, is measured at $0^{\circ}$ on axis, using the beam line in a high spatial resolution - low intensity mode. In this configuration, the beam spot on sample is generally about $1 \mu \mathrm{m}$, but it can go down to a few hundred nanometers [2, 3]. Beam intensity is of a few thousand ions per second, that's to say less than one femtoampere. The second data set comes from emission tomography (Particle Induced X-ray Emission Tomography, PIXET): the beamline has to be used in a higher intensity - larger resolution mode (typically a few hundred picoampere / a few micrometer spot). This is required to obtain enough counting statistics for X-ray emission, following the ionization of sample atoms along the beam path. The analysis of the X-ray energy spectrum gives access to the nature and quantity of emitting atoms. The main advantages of the PIXE technique rely on its sensitivity (down to a few $\mu \mathrm{g} / \mathrm{g}$ ) and its multielementarity. Indeed, all chemical elements of the periodic table can be detected, for a sufficiently high X-ray energy, that's to say typically for $\mathrm{Z}>11$ (Na element), for conventional thin window X-ray detectors. By calculating the Xray production cross section, PIXET reconstruction gives access to the element mass density distribution of each detected chemical element (in $\mu \mathrm{g} / \mathrm{cm}^{3}$ ).

STIMT provides the three dimensional (3D) structure of the sample. More precisely, by calculating the sample's stopping power, quantitative STIMT imaging gives access to the distribution of total mass density (in $\mathrm{g} / \mathrm{cm}^{3}$ ). This information complements PIXET in several aspects, both qualitative and quantitative:

- To help identifying in which structures of interest the X-ray emitting element are localized, keeping in mind that usually STIMT images show better spatial resolution and less noise than PIXET images; 
- To normalize PIXET mass distributions, in order to obtain element concentration distributions (in $\mu \mathrm{g} / \mathrm{g}$ );

- To correct for non linear X-ray production (NLXP), because the ion beam energy decreases into the sample, leading to a variation of ionization cross section;

- To correct for X-ray absorption (XA) within the sample, in the way from the emitting point to the detector.

The Centre d'Etudes Nucléaires de Bordeaux Gradignan (CENBG) develops new methodological approaches including STIMT and PIXET to study the effects of metal oxide nanoparticles in living organisms. Two research topics are explored: (i) nanotoxicology at the level of cells and of small organisms; (ii) optimization of new cancer therapy treatments combining proton-therapy and nanoparticles in a theranostic approach. From a methodological point of view, these topics have motivated the development of non destructive imaging techniques [4] to study cell lines [5-7] and multicellular microorganisms $[8,9]$.

Since the very first experiments, progress in proton beam imaging has been made both for micro- and macroscopic applications. First proton imaging experiments on reference samples [10] a few millimeters in size show better density contrast using proton beams compared to conventional X-ray beams, for a lesser deposited dose. Later on, proton beam microtomography (STIMT and PIXET) was implemented on microscopic samples using protons in the range of a few $\mathrm{MeV}$ [11]. Since then, a wide variety of applications has been developed [1]. The feasibility of PIXET for macroscopic objects has been recently demonstrated at the proton therapy center of Hokkaïdo University Hospital, Japan, on phantom objects 2,2 cm in diameter [12], opening the way to promising studies on small animals for instance. Considering emission and transmission proton tomography data reduction, algorithmic 
developments of data inversion, image registration and data fusion methods may benefit to various research fields, both for micro- and macroscopic applications.

\section{Reconstruction methods for STIMT and PIXET}

Different approaches have been proposed for STIMT and PIXET data reconstruction (review in [8]). All of them rely on algorithms initially designed for medical imaging. Indeed, from a methodological point of view, STIMT could be compared to conventional X-ray scanners. The main difference relies on the calculation of projections. On one hand, X-ray scanners are based on X-ray absorption: projections are obtained by comparing the initial and transmitted number of X-rays. On the other hand, STIMT relies on energy loss: the median (or mean) energy of the protons transmitted through the sample (basically $100 \%$ of the incoming beam) is calculated, which reduces noise in projections. In a similar way, we could compare PIXET to SPECT (Single Photon Emission Computed Tomography). In the first case, photons are emitted along the beam path (projection direction) following the ionization of sample atoms. In the second case, photons are emitted within the complete volume, from the radioactive nuclei marking the molecule of interest. So, for SPECT, the photon signal is attenuated only by absorption processes from the emitting point to the detector, whereas for PIXET both NLXP and XA must be taken into account.

We would like here to give a brief overview of the medical imaging algorithms transferred to ion beam micro-tomography applications. The first STIMT reconstruction codes were based on analytical methods originally designed for X-ray scanners, such as filtered backprojection (FBP) $[13,14]$. Then, algebraic methods such as algebraic reconstruction technique (ART) and statistical methods such as entropy maximum were implemented [15] to reduce noise in the images. Nevertheless, FBP has been generally preferred for its simple, quick and efficient use 
for low noise transmission data, as it relies on the exact mathematical solution of the tomography inversion problem. Indeed STIMT experiments provide enough statistics and are relatively quick (about one hour beam time for a typical 3D $128 \times 128 \times 128$ voxels image). Moreover, transmitted energy projections may be very efficiently filtered, for instance by a classical median filtering applied on the events collected at every pixel, before tomographic reconstruction $[8,16]$.

First attempts to perform STIMT and PIXET data fusion used algebraic methods of Iterative Least Squares Technique (ILST) derived from medical SPECT [11, 17]. However a major simplification was made in these algorithms, by assuming a point-like detector (zero solid angle), whereas most experimental PIXET setups use a very large solid angle in order to reduce acquisition time and/or improve statistics. A development from a medical PET (Positron Emission Tomography) iterative algorithm, taking into account large solid angle, led to the DISRA code (Discrete Image Space Reconstruction Algorithm) [18]. This algorithm has shown to give accurate results on simulated phantoms and experimental reference samples with highly contrasted regions of interest [19]. A quick description of this algorithm may be found in [8]. We would like here to sum up the main difficulties raised by this program:

- As every iteration relies on an analytical inversion method (FBP), the reconstruction is not suited for incomplete (for instance, angle range limited to less than $180^{\circ}$ ) or noisy data sets;

- For low contrasted objects, the reconstruction process converges very slowly and "manual" modification of the convergence factor may be required;

- STIMT and PIXET data are considered all together at each iteration, to generate a tomogram containing the mass fraction distributions of all elements (the total sum of 
all mass fractions is normalized to 1). So, an error that may occur in the quantification or localization of one element for instance would have an influence on all other elements.

Despite these drawbacks, DISRA has remained the most complete reconstruction code designed for STIMT and PIXET and for this reason, has been used and/or modified by several research groups $[20,21,22]$. To overcome the first two difficulties, a major improvement of the code was proposed (JPIXET) by replacing FBP by a method widely used for SPECT imaging, MLEM (Maximum Likelihood Expectation Maximization) [23, 24]. Moreover Graphics Processing Unit (GPU) parallel computing using CUDA (Compute Unified Device Architecture) programming was implemented to fasten the reconstruction process. The MLEM method had been already implemented for PIXET reconstruction by several research groups $[8,25,26]$ but not in a way as complete as JPIXET. Although it is much slower, MLEM may be preferred to FBP for several reasons:

- It produces less noise in the reconstructed image ;

- It can deal with incomplete or noisy data (less projections, limited angle [8]) ;

- As an iterative method, it is more suited to include a correction factor for NLXP and XA.

An accelerated version of MLEM, OSEM (Ordered Subset Expectation Maximization), can be used to accelerate convergence. This method had been implemented in a qualitative version (without mass density calculation) for STIMT and PIXET in the reconstruction code TomoRebuild developed at CENBG [8]. However, as OSEM usually leads to noisier and less contrasted images, MLEM is still preferred. Indeed for microscopic applications, a very rapid reconstruction time is not as drastic as for medical imaging. Moreover, ion beam tomography data sets are still small compared to medical imaging, which reduces reconstruction time. The 
number of data is limited by experimental constraints to images about $128 \times 128 \times 128$ voxels or less, as it takes about 1 hour data acquisition for conventional experimental setups for $3 \mathrm{D}$ STIMT imaging and about $1 \mathrm{~h}$ per slice for PIXET. So, to be able to reduce data acquisition time by reducing the number of projections thanks to MLEM is a very important advantage. $\mathrm{Ng}$ et al. [25] showed the reconstruction of a $30 \times 30$ voxel simple symmetrical object from only 4 projections using this method. Other ways may be explored to reduce acquisition time, such as using multiple detectors for PIXET [review in 1]. We are presenting here a first example of PIXET using 2 detectors.

\section{Experimental procedure}

Caenorhabditis elegans (C. elegans) is a small nematode about $1 \mathrm{~mm}$ in length and $50 \mu \mathrm{m}$ in diameter, usually living in temperate soils and widely used as a model for biological studies (http://www.wormbook.org/). C. elegans populations are cultivated at CENBG and exposed to different environmental conditions to study the biological effect of metal nanoparticles, especially $\mathrm{TiO}_{2}$. The $C$. elegans sample presented here was freeze dried and studied using a 1.5 $\mathrm{MeV}$ proton beam in conditions already presented [9]. In this publication, data reduction was performed with TomoRebuild in the "thin sample" approximation and results were compared to DISRA and JPIXET. We would like here to complete this study and show two major improvements:

- X-ray signal correction for "thick sample", obtained from modeling NLXP and XA, is now included in the reconstruction process;

- Two PIXET detectors were used for this experiment and the fusion of the corresponding reconstructions is presented here.

The two $\mathrm{Si}(\mathrm{Li})$ spectrometer $(\mathrm{e} 2 \mathrm{v}$ Sirius detector, $80 \mathrm{~mm} 2,148 \mathrm{eV}$ resolution for the MnKa line) were placed backwards, symmetrically, at $135^{\circ}$ and at $45^{\circ}$ from the incoming beam (Fig. 
1). The intrinsic efficiency of each detector was determined by previous PIXE analyses of standard samples from Micromatter ${ }^{\mathrm{TM}}$. These standards were thin films of certified thickness (typically $100 \mu \mathrm{g} / \mathrm{cm}^{2}$ ) of $\mathrm{NaCl}, \mathrm{MgF}_{2}, \mathrm{Al}, \mathrm{SiO}, \mathrm{CuSx}, \mathrm{KI}, \mathrm{CaF}_{2}, \mathrm{Ti}, \mathrm{Fe}$, each deposited on a $6.3 \mu \mathrm{m}$ mylar foil.

For STIMT, the beam was scanned over a rectangular area of $170 \mu \mathrm{m}$ in width and $112 \mu \mathrm{m}$ in height, following a grid of 128 pixels in width and 85 pixels in height (i.e. 85 tomographic slices of $128 \times 128$ pixels). The spatial resolution of the reconstructed slices was therefore limited by the scan parameters, to $1.3 \mu \mathrm{m} /$ pixel both in the horizontal and vertical directions. Isolated PIXET slices were probed at selected positions of interested within this STIMT volume. They were chosen from the 3D structure obtained from STIMT reconstruction and also from conventional 2D PIXE analysis within this region. The duration of data acquisition was about $1.5 \mathrm{~h}$ for 3D STIMT and about $2 \mathrm{~h}$ per PIXET slice.

\section{The TomoRebuild software package}

\subsection{General overview}

The TomoRebuild software package has been developed at CENBG for 2D and 3D STIMT and PIXET reconstruction. In its previous version [9], it performed quantitative reconstruction of STIMT and PIXET for "thin samples" (taking into account neither NLXP nor XA), using the GUPIX data base (http://pixe.physics.uoguelph.ca/gupix/main) [27] for the calculation of X-ray emission yields. We present here a new version of this code now available, including "thick sample" correction for NLXP and XA. As in the previous versions, C++ programming with standard libraries was used, in order to avoid any dependency to any graphic library and provide multiplatform use. Optional graphic applications using the ROOT graphic library (https://root.cern.ch/) may be used to display and fit energy spectra for any projection(s). 
However these optional graphic programs were intentionally kept separated from the TomoRebuild code for easier portability.

Data reduction requires different steps which may be run either from a command line instruction or via a user friendly interface, developed as a portable Java plugin in ImageJ. This graphic software package is a public domain, open-source Java-based image processing program developed at the National Institutes of Health (http://rsbweb.nih.gov/ij/). The TomoRebuild ImageJ plugin uses the same text parameter files as the command line version. So all experimental and reconstruction parameters can be easily modified, either directly in the ASCII parameter files using a basic text editor, or via the ImageJ interface. A detailed user guide in English is provided. Sinograms and final reconstructed images are generated in usual binary formats that can be read either using the Amira ${ }^{\circledR}$ graphic library (https://www.fei.com/software/amira-3d-for-life-sciences/) or public domain software packages, such as ImageJ. In this way, any step may be run separately: data sorting for projections calculation, correction of sinograms, "thick sample" correction of X-ray signal (NLXP and XA), tomographic reconstruction (FBP, MLEM, OSEM)... Correction procedures to take into account different spatial resolution and/or small position mismatch between STIMT and PIXET images are included, to improve the fusion of these data. Our aim was to facilitate access to input/output data at each step, so that the user may for instance introduce some homemade procedures if it is required.

Sinogram calculation and options to improve noisy data in TomoRebuild have been already described [8]. PIXET reconstruction is performed separately for each X-ray emitting chemical element to avoid error propagation from one element to another. If the "thin sample" approximation is used (no correction, neither NLXP nor XA) PIXET images may be reconstructed without requiring any STIMT data. STIMT information is required in two cases only: 
- To normalize PIXET element mass distributions $\left(\mu \mathrm{g} / \mathrm{cm}^{3}\right)$ by STIMT local mass content to obtain mass fractions distributions $(\mu \mathrm{g} / \mathrm{g})$;

- To perform NLXA and XP modeling within the 3D STIMT reconstructed image, considering the large PIXET detection solid angle.

\subsection{Reconstruction method}

In this new version of TomoRebuild, MLEM and OSEM methods were improved using optimized methods from the NiftyRec medical imaging library (http://niftyrec.scienceontheweb.net/wordpress/), which is an open source code developed at the Center for Medical Image Computing at University College London [28]. This library was first suggested for PIXET in its GPU version [29]. For TomoRebuild, we used this library in its basic C++ version, to keep easier software package portability. Reconstruction time doesn't constitute a severe limitation. For instance the PIXET slice reconstruction $(128 \times 128$ pixels, 100 projections) displayed in the Results and discussion part, including correction matrix calculation in the STIMT volume $(128 \times 128$ pixels, 100 projections, 85 slices $)$, takes 27 seconds using MLEM on a conventional PC, $1.86 \mathrm{GHz}$ four core Intel Xeon, $32 \mathrm{~Gb}$ DDR4 random access memory, using the Window 7 operating system. NiftyRec algorithm is based on Zeng and Gullberg's projection and backprojection methods [30]. In this method, the reconstructed image is rotated at each projection so that the projection direction (proton beam direction) is always fixed. The image is resampled accordingly at each projection, so that the voxels are always aligned with the projection direction. The correction matrix calculated by TomoRebuild to model NLXP and XA is rotating the same way. For each iteration step, it is multiplied voxel by voxel to the PIXET reconstructed image at each angle (Fig. 2). We could sum up this correction procedure as follows: The correction matrix provides multiplicative 
factors $A_{i}$ that are included in the NiftyRec procedure (MLEM or OSEM). If we call $f$ the density distribution to reconstruct, each projection $p_{j}$ at angle $j$ is obtained from:

$$
p_{j}=\sum_{i=0}^{n-1} R_{i j} A_{i} f_{i}
$$

In this expression, $R$ is the Radon transform operator and $i$ the position index of the voxels in the reconstructed slice of $n$ voxels. The $A_{i}$ correction factors are values between 0 (total attenuation of X-ray signal) and 1 (no attenuation). We should note that TomoRebuild can deal with non-centered projections, that is to say the rotation axis does not have to be at the center of the sinogram [8].

To summarize, the PIXET correction process relies on the correction matrix calculated from the 3D STIMT distribution. However, STIMT and PIXET data are usually obtained from two different experiments. For this reason, it should be carefully checked that there is no position mismatch between the two data sets. The difference in beam positioning that may occur between STIMT and PIXET can be corrected in TomoRebuild by taking into account two shift parameters which are the mismatch in horizontal and vertical directions between the STIMT and PIXET slices, expressed in number of voxels. This procedure was detailed in a previous publication [9] and may be summarized as follows: the STIMT slice(s) are compared to the PIXET slice(s) obtained without correction (so, independently of STIMT). For PIXET, the user may choose any chemical element, since the shift is the same for all of them. The shift parameters are determined "by eye" using an imaging tool permitting the superimposition of the two images with a different color scale and transparency, as, for instance, the free software GIMP (GNU Image Manipulation Program http://www.gimp.org/), as illustrated in [9]. Then, the shift parameters can be entered from the TomoRebuild ImageJ interface or directly in the ASCII parameter file. In a future version of the program, we would 
like to improve this procedure by implementing an image registration algorithm for an automatic determination.

\subsection{Correction matrix}

The correction matrix is calculated once, before PIXET reconstruction, assuming the global composition of the sample is homogeneous (for major chemical elements), which is usually verified for biological specimens. For this, the STIMT slice(s) corresponding to the PIXET slice(s) to reconstruct are identified. NLXP and XA are calculated at each voxel of these(s) STIMT slice(s) and at each projection angle, using numerical functions from the DISRA code [19]. In this code, the large detection solid angle is modeled by dividing the detector entrance window (supposed circular) in surface elements (Fig. 3). XA is calculated along a straight line trajectory (within the complete 3D STIMT image) from every voxel of the slice(s) to every surface element. The total correction matrix $A$ corresponds to the product of NLXP and XA. It is applied on the PIXET slice(s) at each step of the NiftyRec iterative reconstruction. All parameters of this calculation (for instance the number of detector surface elements) may be check and/or modified from the TomoRebuild ImageJ interface or directly in the ASCII parameter file.

\section{Results and discussion}

\subsection{Reconstruction of the C. elegans sample}

We consider STIMT / PIXET data reduction from the C. elegans sample already presented in [9]. Our aim is to extend this study, taking into account X-ray signal correction. Our methodological aim is to compare reconstruction results in various conditions for this type of biological sample: 
- with and without NLXP and XA correction;

- with two X-ray detectors positioned at different angles;

- with different reconstruction algorithms (different methods available in TomoRebuild, DISRA, JPIXET).

All images presented in the following figures were obtained from the AMIRA graphic software package. PIXET reconstruction initially leads to the considered element mass density distribution. The most attenuated reconstructed element, here phosphorus, is displayed in Fig. 4 using MLEM. As expected, the X-ray signal appears attenuated in the regions opposite to the detectors, when comparing reconstructions without / with NLXP and XA correction. This is especially striking at the bottom left side of the sample, which appears very bright (Fig 4a) when facing the X-ray detector (Det. 1, as seen in Fig.1) whereas it is darker for detector 2 (Fig. $4 b$, see arrows). The correction procedure leads to very similar distributions for both detectors (Fig. $4 d-e$ ), with a global increase of about $40 \%$ of mass density values. This result is in good agreement with the decrease of Phosphorus X-ray signal expected for this sample, about $19 \%$ for NLXP and $38 \%$ for XA [9]. Note that a simple fusion (average) of images from detectors 1 and 2 is not sufficient to correct for these effects (Fig. 4c). Only the fusion of corrected images leads to a suitable result (Fig. 4f). We would like to point another methodological point: here the fusion of the element distributions from the two detectors was performed at the end of the MLEM reconstruction. It could be interesting to merge the two distributions within the reconstruction process itself, by multiplying the reconstructed images at each iteration, especially if some voxels are totally attenuated for one detector (this is not the case for this sample). We would like to explore this possibility in further studies. 


\subsection{NLXP and XA correction matrices}

TomoRebuild calculation of NLXP and XA is displayed for the two detectors for two different projection angles: First projection at $0^{\circ}$ (Fig. $5 a$ and $d$ ) and middle projection at $90^{\circ}$ (Fig. $5 b$ and $c$ ). The positions of beam and detectors relative to the image are those depicted in Fig. 1. NLXP correction follows the beam direction (coming horizontal from the left hand side). Calcium NLXP correction appears stronger than phosphorus (comparing Fig. $5 a$ and $d$, left images). This is in agreement with the typical average values of $19 \%$ expected for $\mathrm{P}$ and $28 \%$ for Ca [9]. Obviously NLXP does not depend on the X-ray detector position but only of the orientation of the sample relative to the beam. For this reason, Fig. $5 b$ and c, left images, display strictly identical matrices. On the opposite, XA only depends of the orientation of the sample relative to the detector. This is striking for instance, comparing on one hand Fig. $5 a, b$ (middle images), showing a lighter XA correction at the bottom left side of the sample facing detector 1 and, on the other hand, Fig. $5 c$, where XA correction is lighter at the upper left side, facing detector 2. As expected, XA correction is much smaller for calcium than phosphorus (Fig. $5 a$ and $d$, middle images), following the typical expected average values for this sample: about $38 \%$ for P and $7.5 \%$ for Ca [9]. The combination of NLXP and XA (Fig. $5 a-d$ right hand side images) shows a significant correction effect on the determination of quantitative element mass even for Ca (Fig. $5 d$, right).

\subsection{Mass fraction distributions}

PIXET mass density distributions $\left(\mu \mathrm{g} / \mathrm{cm}^{3}\right)$ are normalized, by dividing, voxel to voxel, the PIXET slice by its corresponding STIMT slice, in order to get mass fractions $(\mu \mathrm{g} / \mathrm{g})$. This procedure requires a careful image treatment before division, to avoid artifacts in the fusion of the two images [9]. For this, the main procedures carried out in TomoRebuild are:

- remove low density noise, especially outside the sample, in the STIMT reconstruction; 
- convolve STIMT image by a Gaussian function in order to get the same spatial resolution as PIXET;

- correct for slight difference in beam positioning between STIMT and PIXET experiments (as summarized in part 4.2).

The final result of PIXET and STIMT data fusion is displayed in Fig. 6 and Tables 1 and 2. The corrected mass fractions distributions applying both NLXP and XA corrections for the reconstructed elements: phosphorus, sulphur, chlorine, potassium, calcium and titanium (following $\mathrm{TiO}_{2}$ nanoparticle uptake by $C$. elegans worms), using NiftyRec MLEM reconstruction are displayed Fig.6. The biological interpretation of such images is beyond the scope of this article. We would like here to compare the quantitative determination of mass fractions between different reconstruction algorithms. Results obtained from TomoRebuild, DISRA and JPIXET have been already compared for "thin sample" reconstruction (without any X-ray signal correction) [9]. We propose here an advance to this study, comparing the new NiftyRec MLEM and OSEM reconstructions implemented in TomoRebuild to FBP and to other reconstruction codes, without correction (Table 1) and including NLXP and XA correction (Table 2). In Table 2 DISRA was implemented both in its original and its GPU accelerated JPIXET versions. Moreover the result of JPIXET MLEM GPU version is also given. Very similar results are obtained whatever the reconstruction algorithm and considered detector. We can note a larger discrepancy for Ti. We could put forward two reasons: (i) Xray signal is lower for Ti compared to the other elements due to lower X-ray production cross section (less statistics), and (ii) the Ti distribution is mostly restricted to a spot, which emphasizes reconstruction and PIXET/STIMT fusion artefacts at the boundaries of this spot. We would like also to point out that, although all algorithms give similar density values, MLEM should be preferred to FBP and OSEM for a better quality of the reconstructed image. FBP intrinsically consists in the discretization of an analytical solution, based on a calculation 
in the Fourier space. For this reason, some negative values appear in the density distribution and sharp boundaries generate high frequency artefacts. Such artefacts have been already mentioned for C. elegans samples [9]. A precise study would require using phantoms of known density and composition, which we intend to do in future work for biological applications. A quantitative study was performed for inertial confinement fusion (ICF) samples using phantoms of regular spherical shape, homogeneous in density and in composition [31]. Deviations up to $\sim 30 \%$ in X-ray counts per pixel were reported, within a 1pixel-thick layer at the inner and outer boundaries of the sample. Such artefacts are known to come from the discretization of the projection data used for FBP reconstruction $[32,33]$ and depend on sample shape, number of projections and number of rays per projection. Indeed, FBP (like all analytical reconstruction methods) relies on the exact continuous mathematical solution of the tomographic reconstruction problem, whereas the projection data are discrete. Calling $\tau$ the pixel width, the maximal spatial frequency that could be accurately reconstructed using FBP is $k_{\max }=1 /(2 \tau)$. However, real tomographic objects are usually not bandlimited to $k_{\max }$. This generates aliasing and interpolation artefacts due to high frequency regions in the sample, especially occurring at sharp edges. Anomalous variations in pixel values (including negative reconstructed values) may be observed in these cases, due to the FBP reconstruction algorithm itself. For instance, a too small number of rays per projection would lead to "streaks" along edges. A too small number of projections would lead to a "star shaped" pattern around the object [32]. These aliasing and interpolation effects may be reduced by using specific filters during FBP reconstruction instead of the basic "ramp" function [33, 5]. For a $n \times n$ pixel slice, Pontau et al. [34] recommended the following data set: beam size equals to pixel size, $n$ projection rays and $(\pi / 2) \times n$ projections. However, in practice, good quality images can be obtained far below this theoretical recommendation (half of this number of projections is more than enough for FBP). Because iterative methods are 
intrinsically discrete, they do not suffer from this bandwidth limitation problem. MLEM is known as one of the most robust algorithms regarding lack of projections [25]. Moreover, as all iterative algorithms, it takes into account that only positive pixel values should be reconstructed. MLEM offers another advantage: as it is based on Poisson distribution, it appears as particularly suited to X-ray emission tomography. We would recommend using its accelerated version, OSEM, as a fast reconstruction test only, as OSEM images appear less contrasted than MLEM.

\section{Conclusion and outlook}

TomoRebuild reconstruction procedure using NiftyRec reconstruction algorithms and DISRA X-ray correction functions for NLXP and XA provides robust and quick quantitative reconstruction of STIMT and PIXET data. The main advantages are:

- The different reconstruction methods available: FBP, MLEM, OSEM;

- The modularity of the different steps of data reduction, from experimental projection data to the final element concentration distributions;

- The independent reconstruction process for STIMT and PIXET data (except for matrix correction and mass fraction calculations);

- The independent reconstruction process for each X-ray emitting chemical element.

Obviously these advantages would not be suitable for samples with unknown and/or heterogeneous composition in major elements. For this type of sample, a complete iterative determination of all X-ray emitting elements combining PIXET and STIMT data at each iteration remains unavoidable, using for instance the DISRA or JPIXET methods. 
TomoRebuild has been developed keeping in mind the wide variety of experimental setups in ion beam analysis laboratories and to overcome the difficulty to deal with sometimes incomplete, noisy or poorly contrasted data. Our concern has been to implement robust reconstruction methods and to give access to all intermediate results, so that every step of the data reduction process may be checked. The experimental results presented here show a good agreement between the different algorithms. A more precise comparative study using numerical and/or experimental phantoms of known composition would permit assessing the accuracy of these methods.

\section{Acknowledgements}

The authors are grateful to Laurent Daudin, Stéphanie Sorieul, Philippe Alfaurt, Franck Delalée and Laurent Sérani, Centre d'Etudes Nucléaires de Bordeaux Gradignan, France, for their support in the technical developments on the beamline. We are grateful to Denis Dupuy, Institut Europeen de Chimie et Biologie, Pessac, France, for providing the C. elegans strain to our laboratory. This work has been financially supported by the CNRS Mission pour l'Interdisciplinarité in the frame of the Défi Imag'In - Proton3D project (CENBG, IMS, LaBRI). Tomography experiments on $C$. elegans have been carried out in the frame of the ANR Program: "Contaminants, Ecosystèmes, Santé 2010" - TITANIUMS project. The Région Nouvelle Aquitaine has supported financially the AIFIRA facility and the technical development of the microbeam line, as also the development of biological protocols through the TOX-NANO program (contract $n^{\circ} 20111201003$ ). This work has been partly supported by the European Community as an Integrating Activity "Support of Public and Industrial Research Using Ion Beam Technology (SPIRIT)" under EC contract $n^{\circ} 227012$ and as an 
"Integrating Activity Supporting Postgraduate Research with Internships in Industry and Training Excellence" (SPRITE) under EC contract n 317169.

\section{References}

[1] C. Michelet, P. Barberet, P. Moretto, H. Seznec, Development and applications of STIM- and PIXE-tomography: A review, Nucl. Instr. Meth. B363 (2015) 55-60.

[2] G. Bench, A. Saint, M. Cholewa, G.J.F. Legge, D.L. Weirup, A.E. Pontau, STIM tomography: a three-dimensional high resolution imaging tool, Nucl. Instr. Meth. B68 (1-4) (1992) 481-490.

[3] N. Gordillo, C. Habchi, L. Daudin, A. Sakellariou, F. Delalée, Ph. Barberet, S. Incerti, H. Seznec, Ph. Moretto, Technical developments for computed tomography on the CENBG nanobeam line, Nuclear Instr. Meth. B 269(20) (2011) 2206-2209.

[4] Q. Le Trequesser, G. Devès, G. Saez, P. Barberet, C. Michelet et al., Single Cell In Situ Detection and Quantification of Metal Oxide Nanoparticles Using Multimodal Correlative Microscopy, Anal. Chem. 86, vol. 15 (2014) 7311-7319.

[5] C. Michelet et $\mathrm{Ph}$. Moretto, Three-dimensional mapping of individual cells using a proton microbeam, Nucl. Instr. Meth. B 150 (1999) 173-178.

[6] Michelet-Habchi et al., 3D Imaging of microscopic structures using a proton beam, IEEE Trans. Nucl. Sci. 52, vol. 3 (2005) 612-617.

[7] C. Habchi et al., Reconstruction of 3D ion beam micro-tomography data for applications in Cell Biology, Nucl. Instr. Meth. B 267, vol. 12/13 (2009) 2107-2112.

[8] C. Habchi, N. Gordillo, S. Bourret, Ph. Barberet, C. Jovet, Ph. Moretto, H. Seznec, Beyond filtered backprojection: A reconstruction software package for ion beam microtomography data, Nucl. Instr. Meth. B 295 (2013) 42-49. 
[9] C. Michelet, P. Barberet, G. Devès, B. Bouguelmouna, S. Bourret, M.-H. Delville, Q. Le Trequesser, N. Gordillo, D.G. Beasley, A.C. Marques, R. Farau, B.R. Toko, J. Campbell, J. Maxwell, Ph. Moretto, H. Seznec, Quantitative reconstruction of PIXETomography data for thin samples using GUPIX X-ray emission yields, Nucl. Instr. Meth. B 348 (2015) 92-99.

[10] M. Cormack, A.M. Koehler, Quantitative proton tomography: preliminary experiments, Phys. Med. Biol. 21 (4) (1976) 560-569.

[11] R.M.S. Schofield, H.W. Lefevre, PIXE-STIM microtomography: zinc and manganese concentrations in a scorpion stinger, Nucl. Instr. Meth. B72 (1992) 104-110.

[12] M. Bazalova-Carter, M. Ahmad, T. Matsuura, S. Takao, Y. Matsuo, R. Fahrig, H. Shirato, K. Umegaki, L. Xing, Proton-induced X-ray fluorescence CT imaging, Med. Phys. 42 (2) (2015) 900-907.

[13] A.E. Pontau, A.J. Antolak, D.H. Morse, A.A. Ver Berkmoes, J.M. Brase, D.W. Heikkinen, H.E. Martz, I.D. Proctor, Ion microbeam tomography, Nucl. Instr. Meth. B 40 (41) (1989) 646-650.

[14] B.E. Fischer, C. Mühlbauer, Microtomography by heavy ions, Nucl. Instr. Meth. B 47 (1990) 271-282.

[15] G. Bench, K.A. Nugent, M. Cholewa, A. Saint, G.J.F. Legge, Submicron STIM tomography reconstruction techniques, Nucl. Instr. Meth. B 54 (1991) 390-396.

[16] J.C. Overley, R.M.S. Schofield, J.D. Macdonald, H.W. Lefevre, Energy-loss image formation in scanning transmission ion microscopy, Nucl. Instr. Meth. B 30 (3) (1988) $337-341$. 
[17] A.J. Antolak, G.S. Bench, PIXE tomography of samples with inhomogeneous elemental composition, Nucl. Instr. Meth. B 88 (1994) 297-307.

[18] A. Sakellariou, M. Cholewa, A. Saint, G.J.F. Legge, An accurate reconstruction algorithm for tomography experiments that involve complex probe-sample interactions, Meas. Sci. Technol. 8 (1997) 746-758.

[19] A. Sakellariou, STIM and PIXE tomography, PhD thesis, School of Physics, University of Melbourne, 2002.

[20] D. Beasley, N.M. Spyrou, 3D quantitative elemental mapping using simultaneous proton induced X-ray emission tomography and scanning transmission ion microscopy tomography, Nucl. Instr. Meth. B 264 (2) (2007) 323-328.

[21] C. Habchi, D.T. Nguyen, Ph. Barberet, S. Incerti, Ph. Moretto, A. Sakellariou, H. Seznec, Reconstruction of 3D ion beam micro-tomography data for applications in Cell Biology, Nucl. Instr. Meth. B 267 (12-13) (2009) 2107-2112.

[22] M. Rothermel, T. Reinert, T. Andrea, T. Butz, First results on ion microtomography at LIPSION, Nucl. Instr. Meth. B 268 (11-12) (2010) 2001-2005.

[23] D.G. Beasley, A.C. Marques, L.C. Alves, R.C. da Silva, Fast simulation of proton induced X-ray emission tomography using CUDA, Nucl. Instr. Meth. B 306 (2013) $109-112$.

[24] D.G. Beasley, L.C. Alves, Ph. Barberet, S. Bourret, G. Devès, N. Gordillo, C. Michelet, Q. Le Trequesser, A.C. Marques, H. Seznec, R.C. da Silva, A comparison of quantitative reconstruction techniques for PIXE-tomography analysis applied to biological samples, Nucl. Instr. Meth. B 331 (2014) 248-252. 
[25] Y.K. Ng, I. Orlic, S.C. Liew, K.K. Loh, S.M. Tang, T. Osipowicz, F. Watt, A PIXE micro-tomography experiment using MLEM algorithm, Nucl. Instr. Meth. B 130 (1-4) (1997) 109-112.

[26] T. Satoh, M. Koka, W. Kada, A. Yokoyama, T. Ohkubo, A. Yamazaki, Y. Ishii, T. Kamiya, N. Kozai, Effectiveness of a combination of ML-EM and STIM-CT in PIXECT for biological specimen, Int. J. PIXE 22 (1-2) (2012) 73-80.

[27] J.L. Campbell, N.I. Boyd, N. Grassi, P. Bonnick, J.A. Maxwell, The Guelph PIXE software package IV, Nucl. Instr. Meth. B 268 (2010) 3356-3363.

[28] S. Pedemonte A. Bousse, K. Erlandsson, M. Modat, S. Arridge, B. F. Hutton, S. Ourselin, GPU accelerated rotation-based emission tomography reconstruction, Proc. IEEE Nucl. Sci. Symp. Med. Imaging Conf. (2010) 2657-2661.

[29] D.G. Beasley, A.C. Marques, L.C.Alves, R.C. da Silva, GPU-accelerated reconstruction methods for Proton Induced X-Ray Emission Tomography, Radiation Physics and Chemistry 95 (2014) 251-253.

[30] G.L Zeng and G.T. Gullberg, Frequency Domain Implementation of the ThreeDimensional Geometric Point Response Correction in SPECT Imaging, IEEE Transactions on Nuclear Science, 39(5) (1992) 1444-1454.

[31] H.Y. Lu, X.D. He, J. Meng, N. Guo, C.C. Rong, W. Zhang, Y.J. Zhang, Z.W. Wang, D.Z. Gao, X.J. Ma, H. Shen, Reconstruction of Ge spatial distribution in ICF target using PIXE-T, Fusion Engineering and Design 113 (2016) 43-50.

[32] A. Rosenfeld, A. Kak. Digital Picture Processing, Second Edition, Volume 1, Academic Press, 1982. 
[33] Peter Toft. The Radon Transform: Theory and Implementation. PhD thesis, Department of Mathematical Modelling, Section for Digital Signal Processing, Technical University of Denmark, 1996. (Direct access : http://petertoft.dk/PhD/)

[34] A.E. Pontau, A.J. Antolak, D.H. Morse, Minimum data set requirements for ion microtomography, Nucl. Instr. Meth. B 54 (1-3) (1991) 383-389.

\section{Table 1}

Reconstruction results for the tomographic slices presented in Fig. 6, as obtained for "thin sample" case, without taking into account X-ray signal attenuation, from different reconstruction methods: TomoRebuild (FBP, NiftyRec MLEM and NiftyRec OSEM) and DISRA (thin sample case). Values marked with an asterisk were taken from [9].

\section{Table 2}

Reconstruction results for the tomographic slices presented in Fig. 6, as obtained for "thick sample" case, taking into account both NLXP and XA, from the three software packages: TomoRebuild (FBP, NiftyRec MLEM and NiftyRec OSEM), DISRA (thick sample case) and JPIXET (DISRA accelerated version and MLEM). Values marked with an asterisk were taken from [9].

\section{Figure captions}

Fig. 1. Configuration of the experimental setup showing the projection direction (beam direction) and the positions of X-ray detectors 1 and 2 relative to the sample, according to the images presented in the following figures. 
Fig. 2. Principle of the correction procedure included in the NiftyRec reconstruction: the correction matrix is rotating in the same way as the sample. As an example, three different orientations are here displayed for phosphorus reconstruction with detector 1: first projection at $0^{\circ}$, middle projection at $90^{\circ}$, last projection at $178.2^{\circ}$ (the projection step is $1.8^{\circ}$ in the $\left[0^{\circ}\right.$; $180^{\circ}[$ interval).

Fig. 3. Principle of the X-ray correction implemented in TomoRebuild, following the DISRA calculation of NLXP along the beam path through the sample, and of XA in the way from the emitting point to the X-ray detector. The large detection solid angle is modeled by dividing the detector into surface elements.

Fig. 4. Element mass density distribution (here of phosphorus) obtained from NiftyRec MLEM reconstruction without (a-c) and with (d-f) X-ray data correction. The effect is particularly conspicuous at the bottom left region of the sample (arrows) facing detector 1 . A linear grey scale was used to code mass density values, ranging from 0 (black) to the maximal indicated density (white). The average value obtained from the total slice (within the sample) is also given for comparison purpose.

Fig. 5. Comparison of the effect of NLXP and XA according to the considered element (here Phosphorus $a-c$ and Calcium $d$ ), to the projection angle (here at $0^{\circ}$ in $a$ and $d$ and at $90^{\circ}$ in $b$ and $c$ ) and to the detector position as described in Fig. 1 ( $a, b, d$ for detector $1 ; c$ for detector 2). A linear grey scale was used to code correction values (between 0 and 1), ranging from the maximal attenuation (black) to no attenuation (white). 
Fig. 6. Final result of PIXET/STIMT fusion: element mass fraction (concentration in $\mu \mathrm{g} / \mathrm{g}$ ) distributions for phosphorus, sulphur, chlorine, potassium, calcium and titanium. Average values for each image are given in Table 2 for comparison purpose, according to the reconstruction algorithm. 